\section{STIMULI-RESPONSIVE MATERIALS}

\section{A memorable reaction}

Angew. Chem. Int. Ed. http://doi.org/f2dqfz (2013)

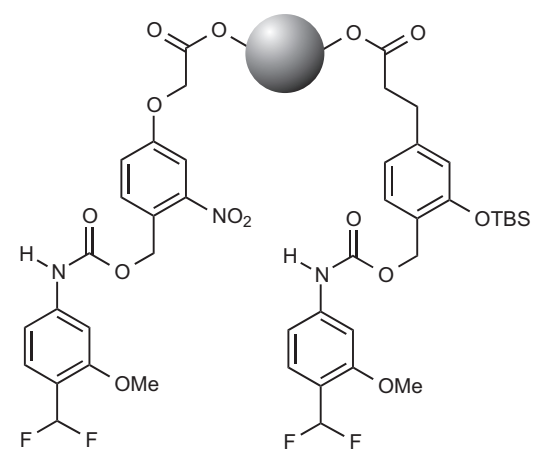

Stimuli-responsive materials have many advantages for constructing mechanically active machines at the micro- and nanoscales. However, although there are many examples of materials that respond to an external stimulus, it is far more difficult to design one that 'remembers' being activated and continues to provide a mechanical function once the stimuli have been removed. Now, Ayusman Sen, Scott Phillips and co-workers at Pennsylvania State University have fabricated a polymer microsphere that acts as a pump once it has been activated by UV light. The chemical reaction that causes the pumping of the fluid surrounding the microsphere continues even after removal of the light.

This polymer sphere is functionalized with two different difluoromethylaryl reagents, one of which breaks down when activated by UV light - releasing several products including fluoride. This fluoride then reacts with the second reagent bound to the polymer, with additional fluoride being released. The two reactions combine to set up a self-propagating autoinductive reaction that amplifies the concentration of fluoride. Once the second reaction is underway, switching off the UV light will terminate the first reaction, but not the second, which continues until all of the secondary reactant is consumed.

The pumping action arises from the formation by the two reactions of a concentration gradient of small-molecule products. The products - a mixture of fluoride ions, protons, carbon dioxide, an aminobenzaldehyde derivative and an organosilyl - diffuse away from the polymer into the fluid. This induces movement of the surrounding fluid towards the microsphere, which the team observed by monitoring the motion of small polystyrene tracer particles. In addition to the continuous pumping behaviour shown by the bifunctionalized microsphere, the team also developed a pump formed from just the UV reactive reagent. This pump could be repeatedly turned on and off by controlling the UV light source.

\section{SINGLE-ATOM CATALYSIS}

\section{Only a supporting role}

J. Am. Chem. Soc. 135, 12634-12645 (2013)

Using smaller particles in heterogeneous catalytic systems is a logical step forward for those trying to achieve greater activity and decrease the amount of expensive metals needed. Amongst other effects, reducing the particle size leads to a higher number of low-coordination active sites per atom of catalyst. Recent improvements in synthesis and characterization techniques have pushed this effort to the extreme, leading to the development of the smallest heterogeneous 'particles' possible - supported singleatom catalysts. As well as better activity, single-atom catalysts could also improve selectivity because, unlike for many other heterogeneous catalysts, they do not suffer from a broad distribution of particle sizes and thus chemistries.

Previous single-atom systems, however, have relied on supports that play an active role in the chemistry, which occurs through the Langmuir-Hinshelwood mechanism whereby the reactants first bind to adjacent sites on the surface before reacting with each other. Now Chaitanya Narula and colleagues at Oak Ridge National Laboratory, Tennessee, have observed the catalytic oxidation of carbon monoxide occurring exclusively on single atoms of platinum supported on inert $\theta$-alumina. The materials were prepared by reacting alumina powder with aqueous chloroplatinic acid, evaporating off the water and pyrolysing the resulting powder. Several samples with varying amounts of platinum were prepared and characterized. Using electron microscopy it was observed that when platinum loading was low $(0.18 \mathrm{wt} \% \mathrm{Pt})$, it existed on the surface as single atoms only, however, when the loading was increased to 1 or $2 \mathrm{wt} \%, 10-20$-atom agglomerates were also observed.

Narula and colleagues confirmed that the supported single atoms of platinum were active for the oxidation of $\mathrm{CO}$ and used density functional theory calculations to investigate the mechanism. Their calculations revealed that, in preference to $\mathrm{CO}$, the bare platinum atoms adsorb $\mathrm{O}_{2}$ (in a side-on geometry). $\mathrm{CO}$ subsequently binds to the oxygenated platinum atom and reacts with the bound $\mathrm{O}_{2}$ to form a carbonate. This species then dissociates to give $\mathrm{CO}_{2}$ and a bound $\mathrm{O}$ atom that can react with a further $\mathrm{CO}$ molecule.

Written by Gavin Armstrong, Stephen Davey, Russell Johnson and Anne Pichon

\section{blog $_{\text {roll }}$ 空}

\section{Sharing your science}

Informal chemistry review articles are a great way to spread the passion you have for your research.

Hours and hours spent in the lab with no results. Finally there's a spike in the spectrum, a precipitate forms, or a colour changes; you've got a result to be proud of, and all of that hard work pays off when you share what you've found with your peers. Whether at a conference, in a peer-reviewed journal, or at a seminar, it's important to communicate the science you're passionate about.

These formal settings are usually what come to mind for sharing your research, but a new blog initiative by Andrew Bissette may also be worth your time. Bissette, who hosts \#chemclub on Twitter and posts round-ups on his blog (http://go.nature.com/LY6Hli), has begun posting an informal review article each month. The first two posts were his own, on protcells (http://go.nature. com/7F7c1G) and the Pummerer reaction (http://go.nature.com/ EmajEr), but since then he has invited others to fill the space with subjects they're familiar with. JesstheChemist, who blogs at The Organic Solution (http://go.nature.com/ARN217) wrote a great review about fluorinated drugs (http://go.nature.com/5Qnoqh).

\#Chemclub Reviews is a new project, but it's a good idea and it will be interesting to watch it develop. Informal short reviews like these are a great way of telling the online chemistry community about the area you work in - a community that is very willing to listen and give relevant input. Reading the reviews is a quick way to learn more about a field that you might not be so familiar with and offers a complementary option to traditional journal review articles. If you would like to contribute an informal review, contact Bissette by e-mail (andrew. bissette@gmail.com) or on Twitter (https://twitter.com/_byronmiller).

Written by Chad Jones, who blogs at http://www.thecollapsedwavefunction.com 